\title{
The Predictive Potential of Elevated Serum Inflammatory Markers in Determining the Need for Intubation in COVID-19 Patients
}

\author{
Samuel Windham*, Kellen Hirsch, Ryan Peterson, David Douin, Lakshmi Chauhan, Lauren \\ Heery, Connor Fling, Nemanja Vukovic, Fernando Holguin, Shanta Zimmer, Kristine \\ Erlandson \\ University of Colorado Anschutz Medical Campus, Denver, CO, USA
}

\begin{abstract}
Introduction: The predictive potential of demographics, clinical characteristics, and inflammatory markers at admission to determine future intubation needs of hospitalised COVID-19 patients is unknown. The study aimed to determine the predictive potential of elevated serum inflammatory markers in determining the need for intubation in COVID-19 Patients. Methods: In a retrospective cohort study of hospitalised SARS-CoV2 positive patients, single and multivariable regression analyses were used to determine covariate effects on intubation odds, and a minimax concave penalty regularised logistic regression was used to build a predictive model. A second prospective independent cohort tested the model. Results: Systemic inflammatory markers obtained at admission were higher in patients that required subsequent intubation, and adjusted odds of intubation increased for every standard deviation above the mean for c-reactive protein (CRP) OR:2.8 (95\% Cl 1.8-4.5, p<0.001) and lactate dehydrogenase OR:2.1 (95\% Cl 1.3$3.3, p=0.002$ ). A predictive model incorporating $\mathrm{C}$-reactive protein, lactate dehydrogenase, and diabetes status at the time of admission predicted intubation status with an area under the curve (AUC) of 0.78 with corresponding sensitivity of $86 \%$, specificity of $63 \%$. This predictive model achieved an AUC of $0.83,91 \%$ sensitivity, and $41 \%$ specificity on the validation cohort. Conclusion: In patients hospitalised with COVID-19, elevated serum inflammatory markers measured within the first twenty-four hours of admission are associated with an increased need for intubation. Additionally, a model of C-reactive protein, lactate dehydrogenase, and the presence of diabetes may play a predictive role in determining the future need for intubation.
\end{abstract}

Keywords: intubation, COVID-19, SARS-CoV-2, inflammatory markers

Received: 15 December 2020 / Accepted: 31 August 2021

\section{INTRODUCTION}

Severe acute respiratory syndrome coronavirus 2 (SARS-CoV-2), and its concomitant clinical disease coronavirus disease 2019 (COVID-19), is an ongoing pandemic originating in China that has quickly spread across the world. As of late October, over 42 million cases and 1.1 million deaths have been reported worldwide [1]. Similar to previous coronavirus infections, patients infected with this novel coronavirus have a variable clinical course. The severity of the disease can vary widely amongst individuals: Fifty-two per cent to eighty-two per cent of patients requiring hospital admission will require supplemental oxygen, and 24-32\% will require intubation [2-6]. Given the variable and possibly unpredictable clinical course of many COV-
ID-19 patients and the potential scarcity of intubation resources worldwide, more data are needed to identify those at greater risk for intubation.

Previous studies have found higher median C-reactive protein, lactate dehydrogenase, neutrophil-to-lymphocyte ratio and $\mathrm{D}$-dimer among hospitalised patients with severe COVID-19 than less severe disease $[7,8]$. Other studies have developed predictive modelling for critical illness as a term encompassing ICU admission, need for intubation, and death on admission $[9,10]$. Regarding triaging admitted patients, one of the essential aspects of critical illness is the need for intubation.

The study aimed to determine the predictive potential of elevated serum inflammatory markers in determining the need for intubation in patients admitted for 
COVID-19, based on admission data. The null hypothesis is that mean serum inflammatory marker levels are not equal for intubated and non-intubated patients.

\section{- METHODS}

A retrospective cohort study was undertaken using the records of the first 158 hospitalised patients aged 18 or older who tested positive for SARS-CoV-2 by nasopharyngeal polymerase chain reaction (PCR) at the University of Colorado Hospital in Aurora, Colorado, between March 19, 2020, and April 2, 2020. Data were collected from the electronic medical record and chart abstraction and recorded in REDCap. Patients were excluded if their records showed they had concomitant viral, bacterial, or fungal co-infections at the time of admission $(n=0)$. The primary outcome was the need for intubation. The institution's criteria for intubation was based on patients who could not maintain $>90 \%$ oxygenation saturation on maximum settings of low-flow oxygen delivery devices. This assessment was carried out by attending staff of the intensive care unit. During the study period, positive pressure ventilation was not utilised due to initial concerns regarding aerosolisation of SARS-CoV-2. Additional outcomes included the need for vasopressor medication, prone positioning, hospital discharge, and mortality on or before April 6, 2020. The second cohort of one hundred and two patients consecutively admitted after April 2, 2020, with the same inclusion criteria, exclusion criteria, and chart data were used to validate the predictive model. The study was reviewed and approved by the Colorado Multiple Institutional Review Board as exempt research.

Categorical and continuous variables were first compared against intubation with Fisher's Exact and Kruskal-Wallace tests, respectively. Then, D-dimer and ferritin were log-transformed. Intubation odds ratios (OR) for covariates of interest are presented as unadjusted and adjusted for a priori confounding variables: age, type 2 diabetes mellitus (DM), symptom duration, ethnicity, and body mass index (BMI). Nonlinear relationships of covariates with intubation were considered using generalised additive models via the $\mathrm{mgcv} \mathrm{R}$ package [11]. (Supplemental online material - Figure A) Time to intubation by C-reactive protein was illustrated and tested via Kaplan-Meier curves and the log-rank test. Multiple imputations by chained equations via the mice $\mathrm{R}$ package [12] was used. In addition, missing data were modelled using random forests and imputed 20 times, and results from the primary analyses were computed and pooled across all imputed data sets.

The minimax concave penalty $(\mathrm{MCP})$ regularised logistic regression [13] was employed to build a sparse predictive model using the covariates and confounders. This procedure fits a series of increasingly saturated models, where saturation is varied using MCP's tuning parameter $\lambda$. A final MCP model indicated covariates most predictive of intubation. The MCP model was tuned via 10 -fold cross-validation (CV) using the ncvreg R package [14]. The optimal model, selected by minimising CV deviance, is presented as a probability formula predicting intubation. The approximate formula was estimated with an MCP-regularized linear probability model. The receiver operating characteristic (ROC) curve and accompanying area under the curve, sensitivity, and specificity were calculated using CV predictions of our optimally tuned models (logistics and linear approximation). The model fitting for each imputed data set was performed to obtain coefficients and took the median coefficient estimate across all imputed data sets to account for missing data. Finally, single imputation was used to represent the figures and CV estimates. The data from the second cohort of 102 patients were plotted on a ROC curve using the predictive model for validating its accuracy. The Significance level was set at 0.05 for all relevant statistical analyses.

\section{DESULTS}

\section{COVID-19 Patient Characteristics}

Of the initial 158 patients admitted and tested positive for SARS-CoV-2 by nasopharyngeal PCR, the mean age (SD) was 56 years (16.8), Table 1. Fifty-one per cent of patients were male, and $49 \%$ were female. Eightyone per cent of patients identified as racial or ethnic minorities. The most prevalent comorbidities were hypertension (56\%), obesity (54\%) and diabetes mellitus (30\%). In addition, patients most commonly presented with cough (82\%), dyspnea (75\%), and fever (74\%). The average duration in days (SD) of symptoms before admission was 6.6 (4.6). Chest x-rays showed bilateral infiltrates in 102 patients (65\%), unilateral infiltrates in 28 patients (18\%), and no evidence of acute pulmonary process in $11 \%$ of patients. 


\section{Hospital Course}

Table 1 shows that 55 (37\%) patients were discharged from the hospital without the need for invasive ventilation, vasopressors, or prone positioning on or before April 6. Sixty-four (41\%) of patients required intubation, $27 \%$ required vasopressor medications, and $15 \%$ were prone positioned during intubation. Four patients (2.5\%) died.

Table 1. Baseline Characteristics of Hospitalised CoVID-19 Patients

\begin{tabular}{|c|c|}
\hline & Overall ( $\mathrm{N}=158$ ) \\
\hline \multicolumn{2}{|l|}{ Clinical Characteristics } \\
\hline Age & $56.2(16.8)$ \\
\hline BMI & $31.3(8.0)$ \\
\hline Male & $81(51.3 \%)$ \\
\hline Active Tobacco Use & $9(5.8 \%)$ \\
\hline White, non-Hispanic & $30(19.5 \%)$ \\
\hline Black & $53(34.4 \%)$ \\
\hline Hispanic & $45(29.2 \%)$ \\
\hline Asian & $11(7.1 \%)$ \\
\hline Other & $15(9.7 \%)$ \\
\hline Diabetes & $47(30.3 \%)$ \\
\hline Hypertension & 87 (56.1\%) \\
\hline COPD & $11(7.1 \%)$ \\
\hline Creatinine $>2 \mathrm{mg} / \mathrm{dL}$ on Admission & $4(2.5 \%)$ \\
\hline Cirrhosis & $3(1.9 \%)$ \\
\hline Coronary Artery Disease & $18(11.6 \%)$ \\
\hline Active Cancer & $11(7.1 \%)$ \\
\hline Immunosuppressed & $10(6.5 \%)$ \\
\hline \multicolumn{2}{|l|}{ Presenting Symptoms } \\
\hline Subjective Fever & $111(74.0 \%)$ \\
\hline Cough & $126(82.4)$ \\
\hline Diarrhoea & $40(26.8 \%)$ \\
\hline Nausea/Vomiting & $42(28.2 \%)$ \\
\hline Myalgia & $34(22.8 \%)$ \\
\hline Dyspnoea & $116(75.3 \%)$ \\
\hline Duration of Symptoms & $6.7(4.6)$ \\
\hline \multicolumn{2}{|l|}{ Chest X-Ray Findings* } \\
\hline Unilateral Infiltrate & $28(17.8 \%)$ \\
\hline Bilateral Infiltrate & $102(65 \%)$ \\
\hline Other Findings & $28(17.8 \%)$ \\
\hline
\end{tabular}

\section{Clinical Course}

Intubation Indicated

$64(40.5 \%)$

Vasopressors Indicated

$42(26.6 \%)$

Prone Positioned

$24(15.2 \%)$

Deceased

$4(2.5 \%)$

Discharge

$55(36.9 \%)$

Data are reported as number (\%) or mean (standard deviation). * Data collected within 24 hours of hospital admission. $\mathrm{BMI}=$ body mass index, $\mathrm{COPD}=$ chronic obstructive pulmonary disease .

\section{Characteristics of Intubated Patients}

Oxygen requirements, clinical characteristics, and P: F ratio at an interval of eight hours are shown in Table 2. The average SOFA score on intubation was 4.5 (SD 2.1), patients were intubated for 11.9 (SD 8.5) days, and one patient was put on non-invasive ventilation before intubation. The average P: F ratio on intubation was 146.3 (SD 84.1) in the initial eight hours from intubation and 219.9 (SD 81.4) at 72 hours from intubation.

\section{Differences between Intubated and Non-Intubated Patients}

Table 3 shows no significant differences in age, body mass index (BMI), or ethnicity between patients with or without intubation. Intubated patients were more likely to have diabetes mellitus ( 43 vs $22 \%, \mathrm{p}=0.012$ ), but not other comorbidities.

Intubated patients had a similar duration of symptoms but were less likely to report nausea or vomiting ( 16 vs $36 \%, \mathrm{p}=0.014$ ) or diarrhoea ( 18 vs $32 \%, \mathrm{p}=0.06$ ). No other differences in presenting symptoms were observed between the two groups.

Intubated patients were more likely to have bilateral lung infiltrates on admission chest X-ray (81 vs $54 \%$, $\mathrm{p}<0.001)$. In contrast, unilateral lung infiltrates were seen in $22 \%$ of the non-intubated group vs $11.1 \%$ of the intubated group $(\mathrm{p}=0.09)$.

Differences in systemic inflammatory markers among patients that did or did not require intubation are shown in Table 3. Compared to non-intubated patients, those requiring intubation had significantly higher C-reactive protein (mean $=70.3 \mathrm{mg} / \mathrm{L}$ vs $142.5 \mathrm{mg} / \mathrm{L}$, $\mathrm{p}<0.001)$ and lactate dehydrogenase $(311.5 \mathrm{U} / \mathrm{L}$ vs 415.4 $\mathrm{U} / \mathrm{L}, \mathrm{p}<0.001)$. Ferritin, D-dimer, and the neutrophillymphocyte ratio were also significantly higher in intubated patients $(\mathrm{p}=0.08, \mathrm{p}=0.03, \mathrm{p}<0.001$, respectively) (Figure A). Therefore the null hypothesis is upheld.

The effect of inflammatory markers and other clinical predictors were evaluated on the odds for intubation, before and after adjusting for confounding variables such as age, race or ethnicity, sex, BMI, diabetes, and symptom duration). As shown in Table 4 and Supplemental online material - Figure B, in univariate models, higher C-reactive protein, lactate dehydrogenase, Ddimer, neutrophil-lymphocyte ratio, diabetes mellitus, and bilateral infiltrate on chest $\mathrm{x}$-ray were associated with greater odds of requiring intubation. After adjusting, the odds of intubation were significantly greater as inflammatory markers increased, per standard devia- 
tion increase in C-reactive protein (OR 2.81), lactate dehydrogenase (OR 2.10), and neutrophil-lymphocyte ratio (OR 2.21). For example, for every increase of 78 $\mathrm{mg} / \mathrm{L}$ in C-reactive protein, the odds of intubation are multiplied by 2.81 . We did not observe a significant effect of age, sex, ethnicity, BMI, symptom duration, ferritin, or D-dimer on the odds for intubation. Collinearity among variables was also explored (Supplemental online material - Table A).

In a sensitivity analysis, the effect of each covariate on the odds for intubation for patients that were intubated at least 24 hours after admission $(n=23)$ versus those not requiring intubation $(\mathrm{n}=94)$ were explored. C-reactive protein, lactate dehydrogenase, and bilateral

Table 2. Characteristics of Intubated CoVID-19 Patients

\begin{tabular}{|c|c|}
\hline & Overall ( $N=64)$ \\
\hline \multicolumn{2}{|l|}{ Clinical Characteristics } \\
\hline $\begin{array}{l}\text { Oxygen Requirement on Admission in } \\
\text { Litres of Oxygen }\end{array}$ & $5.6(13.2)$ \\
\hline $\begin{array}{l}\text { Highest Oxygen Requirement Stable } \\
>1 \text { Hour within } 24 \text { Hours of Admission } \\
\text { in Litres of Oxygen }\end{array}$ & $5.0(4.1)$ \\
\hline $\begin{array}{l}\text { Use of Non-Invasive Ventilation Before } \\
\text { Intubation }\end{array}$ & $1(1.6 \%)$ \\
\hline $\begin{array}{l}\text { Oxygen Requirement within one hour } \\
\text { of Intubation in Litres of Oxygen }\end{array}$ & $12.9(12.7)$ \\
\hline SOFA on Intubation & $4.5(2.1)$ \\
\hline Days Intubated & $11.926(8.519)$ \\
\hline \multicolumn{2}{|l|}{ PaO2 to Fi02 at 8 Hour Intervals } \\
\hline P:F Ratio at $8 \mathrm{~h}$ & $146.3(84.1)$ \\
\hline$P: F$ Ratio at $16 \mathrm{~h}$ & $194.9(69.1)$ \\
\hline P:F Ratio at $24 \mathrm{~h}$ & $204.0(63.4)$ \\
\hline P:F Ratio at $32 \mathrm{~h}$ & $216.8(65.5)$ \\
\hline P:F Ratio at $40 h$ & $216.2(65.9)$ \\
\hline P:F Ratio at $48 \mathrm{~h}$ & $204.7(75.3)$ \\
\hline P:F Ratio at $56 \mathrm{~h}$ & $212.5(73.9)$ \\
\hline P:F Ratio at $64 \mathrm{~h}$ & $217.0(71.2)$ \\
\hline P:F Ratio at $72 \mathrm{~h}$ & $219.9(81.4)$ \\
\hline \multicolumn{2}{|l|}{ Pa02 to (Fi02* PEEP) at 8 Hour Intervals } \\
\hline P:FP Ratio at 8h & $12.3(7.4)$ \\
\hline P:FP Ratio at $16 \mathrm{~h}$ & $17.3(9.8)$ \\
\hline P:FP Ratio at $24 \mathrm{~h}$ & $17.5(8.0)$ \\
\hline P:FP Ratio at $32 \mathrm{~h}$ & $19.1(8.8)$ \\
\hline P:FP Ratio at 40h & $20.1(10.8)$ \\
\hline P:FP Ratio at $48 \mathrm{~h}$ & $18.2(13.7)$ \\
\hline P:FP Ratio at $56 \mathrm{~h}$ & $19.1(9.5)$ \\
\hline P:FP Ratio at $64 \mathrm{~h}$ & $20.6(12.8)$ \\
\hline P:FP Ratio at $72 \mathrm{~h}$ & $21.2(13.0)$ \\
\hline
\end{tabular}

Data are reported as number (\%) or mean (standard deviation). $\mathrm{h}=$ hour, Pa02 $=$ Arterial oxygen content, $\mathrm{FiO2}=$ fractional of inspired oxygen, $\mathrm{PEEP}=$ Positive end expiratory pressure, $\mathrm{P}: \mathrm{F}=\mathrm{Pa} 02 /$ $\mathrm{FiO2}, \mathrm{P}: \mathrm{FP}=\mathrm{PaO} 2 /\left(\mathrm{FiO2}{ }^{*} \mathrm{PEEP}\right)$ infiltrate on chest $\mathrm{x}$-ray remained significantly associated with later intubation, compared to no intubation, with an unadjusted odds ratio of 2.24 (95\% CI 1.5, 3.4, $\mathrm{p}<0.001$ ), OR 1.87 (95\% CI 1.2, 2.9, $\mathrm{p}=0.006)$, and OR 3.37 (95\% CI 1.3, 8.5, p=0.01), respectively (Supplemental online material - Table B).

\section{Characterisation of Patients by Serum C-reactive protein Concentration and Probability of Intubation Over Time by Serum C-reactive protein Concentra- tion}

To understand the role of low versus high C-reactive protein in clinical characteristics and outcomes, patients were stratified by a C-reactive protein concentration of $\leq 100 \mathrm{mg} / \mathrm{L}$ versus higher than $100 \mathrm{mg} / \mathrm{L}$ (Supplemental online material - Table C). Patients with higher C-reactive protein were more likely to present with cough $(p=0.027)$ and bilateral lung infiltrates on chest $\mathrm{x}$-ray $(\mathrm{p}=0.009)$, while those with low C-reactive protein were more likely to present with nausea/ vomiting $(\mathrm{p}=0.022)$ and have a negative chest $\mathrm{x}$-ray $(p=0.016)$. No other significant differences were detected in presenting characteristics by C-reactive protein stratification. However, as shown in the Kaplan-Meier curve Figure 1, the probability of intubation over time was significantly greater in the high versus low $\mathrm{C}$-reactive protein group $(\mathrm{p}<0.0001)$, with more than half of patients in the high C-reactive protein group intubated within the first two days of admission.

\section{Generating a Predictive Model for Probability of Intubation}

Using a sparse predictive model using the covariates of interest (C-reactive protein, lactate dehydrogenase, $\mathrm{D}$ dimer, ferritin, age, BMI, non-white, diabetes mellitus, male, symptom duration before admission, and bilateral chest $\mathrm{x}$-ray infiltrates), C-reactive protein, lactate dehydrogenase and diabetes mellitus were selected by the model as the most predictive factors for predicting the need for intubation. These three variables correctly classified intubated status $71 \%$ of the time, corresponding to a sensitivity of $86 \%$, specificity of $63 \%$, positive predictive value (PPV) of $61 \%$, negative predictive value (NPV) of $87 \%$ using a $30 \%$ threshold. The area under the ROC curve (AUC) was 78\% (Figure 2).

Bilateral infiltrates on chest x-ray added marginally to the model's predictive performance (data not shown) but was removed as it resulted in a decrease in model sensitivity. The predictive model was then ap- 
Table 3. Characteristics of Hospitalised CoVID-19 Patients Stratified by Intubation Status

\begin{tabular}{|c|c|c|c|c|}
\hline & $\begin{array}{l}\text { Not Intubated } \\
\qquad(N=94)\end{array}$ & $\begin{array}{l}\text { Intubated } \\
(N=64)\end{array}$ & $\begin{array}{c}\text { N-Missing } \\
\text { (Not intubated/ Intubated) }\end{array}$ & p-value \\
\hline \multicolumn{5}{|l|}{ Clinical Characteristics } \\
\hline Age & $55.36(17.33)$ & $57.32(15.90)$ & $0 / 1$ & 0.415 \\
\hline BMI & $30.85(7.84)$ & $32.01(8.2)$ & $5 / 0$ & 0.436 \\
\hline Male & 45 (47.9\%) & $36(56.2 \%)$ & NA & 0.333 \\
\hline Active Tobacco Use & $4(4.3 \%)$ & $5(8.1 \%)$ & NA & 0.485 \\
\hline $\begin{array}{l}\text { Non-White Race and/or Hispanic } \\
\text { Ethnicity }\end{array}$ & $71(76.3 \%)$ & $53(86.9 \%)$ & NA & 0.145 \\
\hline Diabetes & $21(22.3 \%)$ & $26(42.6 \%)$ & NA & 0.012 \\
\hline Hypertension & $52(55.3 \%)$ & $35(57.4 \%)$ & NA & 0.869 \\
\hline COPD & $6(6.4 \%)$ & $5(8.2 \%)$ & NA & 0.753 \\
\hline Creatinine $>2 \mathrm{mg} / \mathrm{dL}$ on Admission & $1(1.1 \%)$ & $3(4.8 \%)$ & NA & 0.303 \\
\hline Cirrhosis & $1(1.1 \%)$ & $2(3.3 \%)$ & NA & 0.562 \\
\hline Coronary Artery Disease & $12(12.8 \%)$ & $6(9.8 \%)$ & NA & 0.620 \\
\hline Active Cancer & $6(6.4 \%)$ & $5(8.2 \%)$ & NA & 0.753 \\
\hline Immunosuppressed & $8(8.6 \%)$ & $2(3.3 \%)$ & NA & 0.317 \\
\hline \multicolumn{5}{|l|}{ Presenting Symptoms } \\
\hline Subjective Fever & $72(77.4 \%)$ & $39(68.4 \%)$ & NA & 0.253 \\
\hline Cough & $75(79.8 \%)$ & $51(86.4 \%)$ & NA & 0.385 \\
\hline Diarrhoea & $30(32.3 \%)$ & $10(17.9 \%)$ & NA & 0.059 \\
\hline Nausea/Vomiting & $33(35.5 \%)$ & $9(16.1 \%)$ & NA & 0.014 \\
\hline Myalgia & $20(21.5 \%)$ & $14(25 \%)$ & NA & 0.688 \\
\hline Dyspnoea & $74(78.7 \%)$ & $42(70 \%)$ & NA & 0.253 \\
\hline Duration of Symptoms & $6.7(4.53)$ & $6.57(4.81)$ & $1 / 6$ & 0.796 \\
\hline \multicolumn{5}{|l|}{ Chest X-Ray Findings* } \\
\hline Clear & $12(13.8 \%)$ & $4(6.3 \%)$ & NA & 0.192 \\
\hline Unilateral Infiltrate & $21(22.3 \%)$ & $7(11.1 \%)$ & NA & 0.090 \\
\hline Bilateral Infiltrate & $51(54.3 \%)$ & $51(81 \%)$ & NA & $<0.001$ \\
\hline Pleural Effusion & $0(0 \%)$ & $2(3.2 \%)$ & NA & 0.159 \\
\hline Other Finding & $6(6.4 \%)$ & $3(4.8 \%)$ & NA & 0.741 \\
\hline \multicolumn{5}{|l|}{ Laboratory Findings* } \\
\hline CRP mg/L & $70.34(60.5)$ & $142.50(83.36)$ & $2 / 6$ & $<0.001$ \\
\hline $\mathrm{LDH} U / \mathrm{L}$ & 311.53 (102.3) & $415.37(160.22)$ & $5 / 13$ & $<0.001$ \\
\hline D-dimer FEU & $1199.23(1692.86)$ & $4475.20(14140.16)$ & $15 / 14$ & 0.033 \\
\hline Ferritin $\mathrm{ng} / \mathrm{mL}$ & $428.47(426.81)$ & 790.62 (1236.12) & $7 / 17$ & 0.079 \\
\hline Neutrophils x10^9/L & $4.55(2.11)$ & $7.20(3.68)$ & $59 / 24$ & $<0.001$ \\
\hline Lymphocytes x10^9/L & $3.60(14.03)$ & $1.08(0.63)$ & $59 / 24$ & 0.067 \\
\hline NLR & $4.20(2.41)$ & $8.33(6.51)$ & $59 / 24$ & $<0.001$ \\
\hline \multicolumn{5}{|l|}{ Clinical Course } \\
\hline Deceased & $2(2.1 \%)$ & $2(3.1 \%)$ & NA & 1.00 \\
\hline Discharged & $53(57.6 \%)$ & $2(3.5 \%)$ & NA & $<0.001$ \\
\hline
\end{tabular}

plied to the separate 102 patient validation cohort data whose descriptive data are listed in Supplemental online material - Table D, which showed a sensitivity of $91 \%$, specificity of $41 \%$, PPV of $44 \%$, and NPV of $90 \%$ at the $30 \%$ threshold. The prediction model diagnostic accuracy with alternative prediction thresholds are shown in Supplemental online material - Table E.

\section{Discussion}

A retrospective cohort study characterised the demographics, comorbidities, presenting symptoms, and 

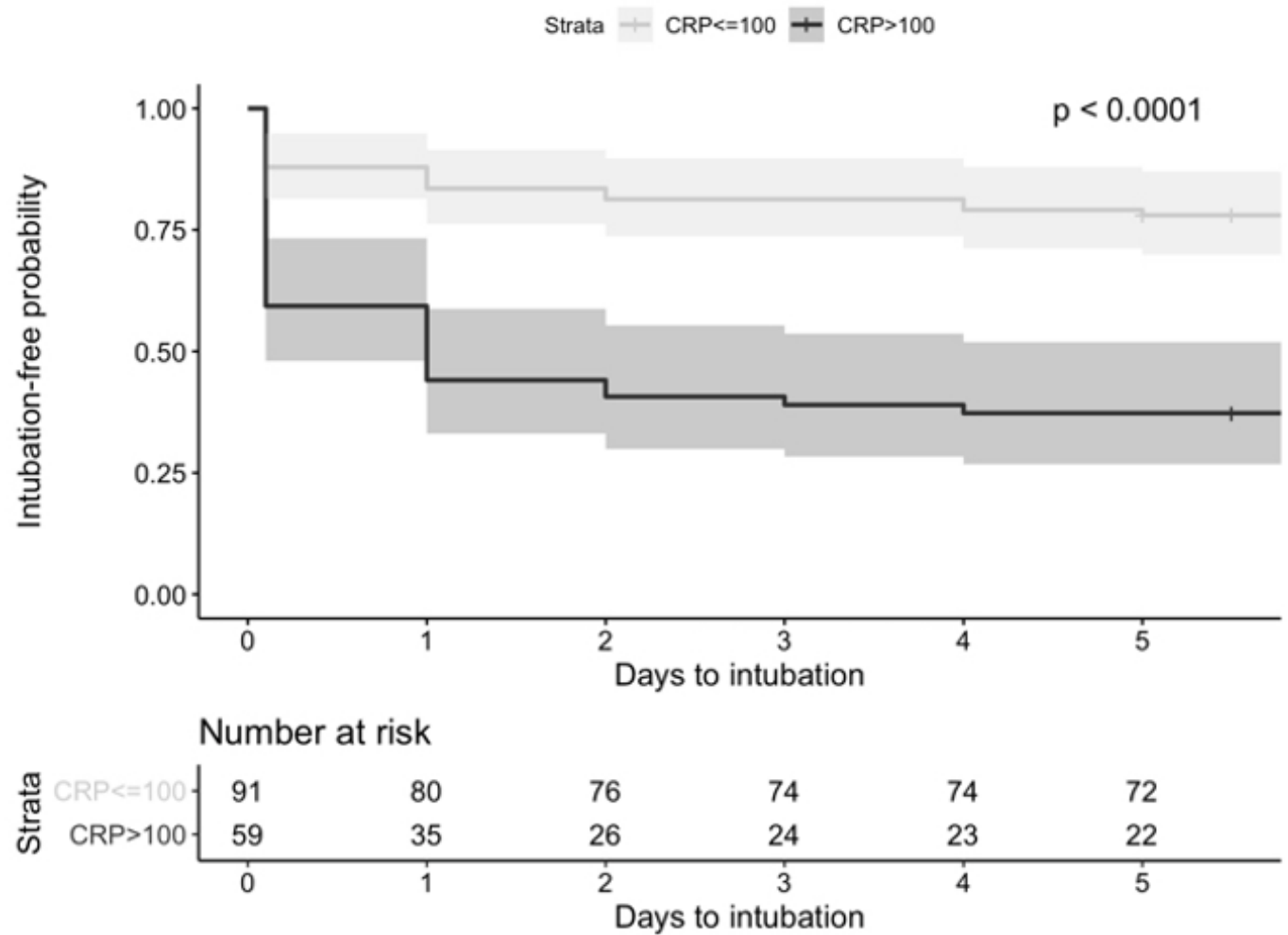

Fig.1. Kaplan-Meier curves for intubation-free survival of hospitalized CoVID-19 patients stratified by C-reactive protein (CRP). Patients with low CRP ( $<100 \mathrm{mg} / \mathrm{L}$ ) have a significantly higher probability of iuntubation-free survival, as compared to patients with high CRP $(\geq 100 \mathrm{mg} / \mathrm{L})(\mathrm{p}<0.001)$

clinical findings of the first 158 patients admitted with positive SARS-CoV-2 at a large tertiary care institution. In addition, a variety of risk factors for intubation in these hospitalised patients, including diabetes mellitus, bilateral infiltrate on chest x-ray, and elevated inflammatory markers were identified.

In a predictive model, a high $\mathrm{C}$-reactive protein, lactate dehydrogenase and presence of diabetes melli- tus predicted the need for intubation, with high sensitivity and moderate specificity. These findings were replicated in a separate validation cohort, revealing a similarly high sensitivity and moderate specificity, key characteristics for a screening test where a more cautious approach is desired. The initial C-reactive protein on admission was predictive of subsequent risk for intubation.

Table 4. Covariate Effects on Odds of Intubation

\begin{tabular}{|c|c|c|c|c|c|c|}
\hline \multirow{2}{*}{ Term } & \multicolumn{3}{|c|}{ Unadjusted } & \multicolumn{3}{|c|}{ Adjusted* } \\
\hline & OR & $95 \% \mathrm{Cl}$ & p-value & OR & $95 \% \mathrm{Cl}$ & p-value \\
\hline 1 SD change in CRP $(78.0 \mathrm{mg} / \mathrm{L})$ & 3.05 & $(1.9,4.8)$ & $<0.001$ & 2.81 & $(1.8,4.5)$ & $<0.001$ \\
\hline 1 SD change in LDH (135.6 U/L) & 2.31 & $(1.5,3.6)$ & $<0.001$ & 2.10 & $(1.3,3.3)$ & 0.002 \\
\hline 1 SD change in Log D-Dimer (0.94 log FEU) & 1.47 & $(1,2.2)$ & 0.048 & 1.33 & $(0.8,2.1)$ & 0.22 \\
\hline 1 SD change in NLR (5.4) & 1.94 & $(1.1,3.5)$ & 0.031 & 2.21 & $(1.1,4.5)$ & 0.033 \\
\hline $1 \mathrm{SD}$ change in Log Ferritin (1.12 log ng/mL) & 1.45 & $(1,2.1)$ & 0.055 & 1.29 & $(0.9,1.9)$ & 0.23 \\
\hline $1 \mathrm{SD}$ change in BMI $\left(8.0 \mathrm{~kg} / \mathrm{m}^{2}\right)$ & 1.15 & $(0.8,1.6)$ & 0.39 & 1.07 & $(0.7,1.6)$ & 0.71 \\
\hline 1 SD change in age (16.8 years) & 1.13 & $(0.8,1.6)$ & 0.45 & 1.15 & $(0.8,1.7)$ & 0.48 \\
\hline Symptom Duration & 0.99 & $(0.9,1.1)$ & 0.86 & 0.99 & $(0.9,1.1)$ & 0.72 \\
\hline DM & 2.52 & $(1.3,5.1)$ & 0.010 & 1.98 & $(0.9,4.3)$ & 0.081 \\
\hline Ethnicity/Race is Non-White & 2.08 & $(0.9,5)$ & 0.10 & 1.96 & $(0.8,5.1)$ & 0.17 \\
\hline Bilateral Infiltrate on X-Ray & 3.6 & $(1.7,7.6)$ & 0.001 & 3.39 & $(1.5,7.6)$ & 0.003 \\
\hline
\end{tabular}




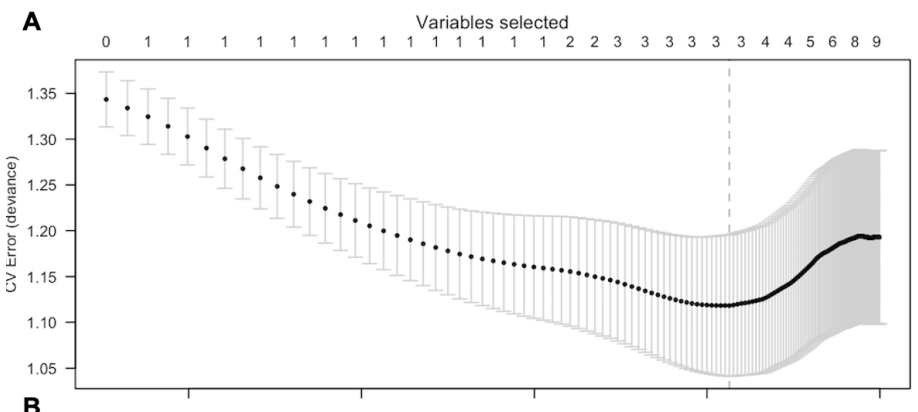

C
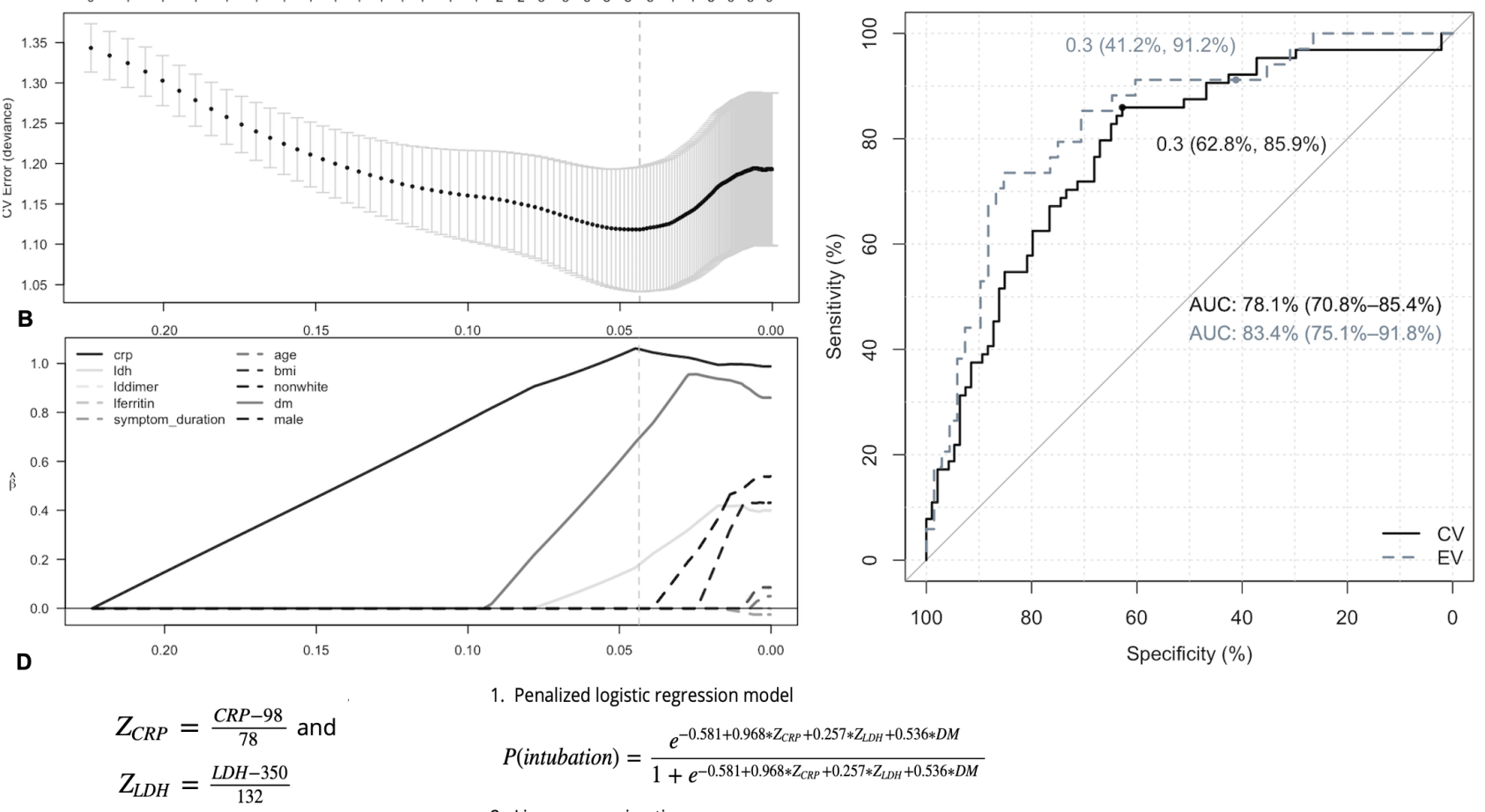

1. Penalized logistic regression model

$$
\begin{gathered}
P(\text { intubation })=\frac{e^{-0.581+0.968 * Z_{C R P}+0.257 * Z_{L D H}+0.536 * D M}}{1+e^{-0.581+0.968 * Z_{C R P}+0.257 * Z_{L D H}+0.536 * D M}} \\
\text { 2. Linear approximation } \\
P(\text { intubation })=0.378+0.207 * Z_{C R P}+0.023 * Z_{L D H}+0.09 * D M
\end{gathered}
$$

Fig. 2. Predictive model for intubation and mechanical ventilation fit via the Minimax Concave Penalty. (A) Cross-validation deviance indicates optimal model has 3 predictors, including (B) C-reactive protein (CRP), lactate dehydrogenase (LDH), and type 2 diabetes mellitus as most predictive covariates. (C) Cross validated ROC curve of logistic model (CV) along with ROC curve on the validation cohort (EV). (D) Optimal model shown as probability formula where coviariates are centered and scaled using observed mean and standard deviation. DM signifies patient has type 2 diabetes (yes=1, no $=0$ ). Both logistic regression and linear approximation formulas presented.

There is a need to quickly identify and triage highrisk patients with COVID-19 who are likely to require intubation, both for appropriate patient triage and management of limited resources [15]. The described derived model is easy to apply and utilises commonly available laboratory values that have biologically plausible links to the disease process and clinical history previously shown to be a risk factor for intubation in pneumonia [16]. It provided good sensitivity for risk for intubation when used in a cohort with many similarities, although our validation cohort did require more prone positioning and had more deceased patients despite having more patients reach discharge in the specified time frame. Given the strain on resources COVID-19 represents, it will be critically important to identify where resources will need to be used as soon as possible. The present findings that three simple measures, high C-reactive protein, lactate dehydrogenase, and presence of diabetes mellitus, can quickly identify those at highest risk for intubation can augment clini- cal findings in identifying patients that may necessitate intensive care unit admission.

Previous studies have examined the predictive qualities of inflammatory markers in COVID-19, although to a different degree than this study. In a retrospective study, Barrett et al. (2021), a cohort of 1123 patients with COVID-19 were analysed for initial laboratory values and severity of illness, including death, intubation, need for renal replacement therapy, and ICU admission [17]. Univariate analysis found statistically significant, but weak links between elevated C-reactive protein and lactate dehydrogenase and the need for intubation with Spearman's $\mathrm{p}^{2}$ reported as 0.090 and 0.108 , respectively. A C-reactive protein of $>8 \mathrm{mg} / \mathrm{L}$ showed $100 \%$ sensitivity and negative predictive value for intubation when variables were dichotomised to normal or above normal. The specificity and positive predictive value were poor for C-reactive protein at $4 \%$ and $27 \%$.

Compared to the dichotomised approach taken by 
Barret et al. (2021), the relationship between intubation and inflammatory markers as continuous and categorical variables in the analysis was tested. This allowed us to explore higher levels of inflammatory markers as predictors of severe disease. Ultimately, a cut-off for C-reactive protein of $100 \mathrm{mg} / \mathrm{L}$ in our final predictive model, which was higher than the cut-off of $8 \mathrm{mg} / \mathrm{dl}$ used in the Barrett et al. (2021) study, was used. The population studied by Barrett et al. was dissimilar to the population in the current study, with higher patients with chronic kidney disease, hypertension, coronary artery disease, and diabetes [17]. These differences in population, approach, and analysis may account for the differing predictive ability noted. Moving forward, the validity of this predictive model should be tested in large populations with different demographics and comorbidities.

In line with previous data regarding the severity of disease and gastrointestinal symptoms, gastrointestinal symptoms such as nausea, vomiting or diarrhoea were negatively correlated with the need for intubation [10]. Other studies have found no link between mortality, need for intubation, nor severe disease $[18,19]$. Thus, it is not clear whether potential gastrointestinal tract infection is protective for intubation or if this is due to reporting bias; it is an area that warrants more rigorous clinical and pre-clinical investigation.

In the current study, the ideal timing for intubation was not defined in COVID-19, and individual providers could have different thresholds for intubation that would affect which patients experienced our primary outcome. This ideal timing has not been defined in COVID-19, and individual providers could have different thresholds for intubation. To address this limitation, data on oxygenation status pre-intubation and 72 hours post-intubation was collected. Intubated patients, on average, required 12.9L of oxygen before intubation. Based on the updated and revised version of the "Berlin Definition" of the acute respiratory distress syndrome (ARDS), the intubated patients had, on average, moderate severity acute respiratory distress syndrome on intubation that eventually improved to mild after 72 hours [20]. The average length of intubation was 11.9 days. All of this data shows that despite different intubation patterns, the intubated patients included in this study showed a reasonable level of hypoxemia to warrant intubation.

As a single-centre study, the current findings may lack generalizability. The measurement of C-reactive protein was at a single point at admission, and the change in C-reactive protein over time may be more informative as to the need for intubation. However, the stated goal was to understand the utility of this initial assessment. It is accepted that symptoms were self-reported and may not be a reliable indicator of the full symptomology in the cohort, especially given the rapidity with which some patients were intubated. As reported in patients' notes, laboratory data and imaging were obtained through routine clinical care, driven by clinical decision making, and have undoubtedly contributed to missing data. Moreover, the final evaluation of the primary endpoint was performed four days following the last admission and may have missed further intubations or clinical changes.

\section{CONCLUSION}

In patients hospitalised with COVID-19, high initial Creactive protein was independently associated with the need for intubation and, in conjunction with high lactate dehydrogenase and diabetes mellitus, was strongly predictive of intubation. However, further investigation using larger, multicentre cohort studies are needed to validate the relationship described in this study.

\section{ACKNOWLEDGEMENTS}

We appreciate the Pulmonary Translational Research Core (PTraC) contributions and their support in statistical analysis.

\section{CONFLICT OF INTEREST}

None to declare.

\section{DEFERENCES}

1. Organisation WH. COVID-19 Weekly Epidemiological Update. 2020.

2. Guan WJ, Ni ZY, Hu Y, et al. Clinical Characteristics of Coronavirus Disease 2019 in China. N Engl J Med. 2020 Apr; 58(4): 711-712.

3. Wu C, Chen X, Cai Y, et al. Risk Factors Associated With Acute Respiratory Distress Syndrome and Death in Patients With Coronavirus Disease 2019 Pneumonia in Wuhan, China. JAMA Intern Med. 2020 Jul 1;180(7):1031.

4. Zhang JJ, Dong X, Cao YY, et al. Clinical characteristics of 140 patients infected with SARS-CoV-2 in Wuhan, China. Allergy. $2020 \mathrm{Jul} ; 75(7): 1730-1741$.

5. Guo T, Fan $\mathrm{Y}$, Chen $\mathrm{M}$, et al. Cardiovascular Implications of 
22 - The Journal of Critical Care Medicine 2022;8(1)

Fatal Outcomes of Patients With Coronavirus Disease 2019 (COVID-19). JAMA Cardiol. 2020 Jul 1;5(7):811-818. Erratum in: JAMA Cardiol. 2020 Jul 1;5(7):848.

6. Zhou F, Yu T, Du R, et al. Clinical course and risk factors for mortality of adult inpatients with COVID-19 in Wuhan, China: a retrospective cohort study. Lancet. 2020;395(10229):10541062.

7. Wang $\mathrm{D}, \mathrm{Hu} \mathrm{B}, \mathrm{Hu} \mathrm{C}$, et al. Clinical Characteristics of 138 Hospitalised Patients With 2019 Novel Coronavirus-Infected Pneumonia in Wuhan, China. Jama. 2020 Mar 17;323(11):10611069. Erratum in: JAMA. 2021 Mar 16;325(11):1113.

8. Qin C, Zhou L, Hu Z, et al. Dysregulation of immune response in patients with COVID-19 in Wuhan, China. Clin Infect Dis. 2020 Mar 12: ciaa248.

9. Schalekamp S, Huisman M, Dijk RAv, et al. Model-based Prediction of Critical Illness in Hospitalised Patients with COVID-19. Radiology.0(0):202723.

10. Ji D, Zhang $D, X u J$, et al. Prediction for Progression Risk in Patients With COVID-19 Pneumonia: The CALL Score. Clinical Infectious Diseases. 2020;71(6):1393-1399.

11. Wood SN. Fast stable restricted maximum likelihood and marginal likelihood estimation of semiparametric generalised linear models. Journal of the Royal Statistical Society: Series B (Statistical Methodology). 2011;73(1):3-36.

12. van Buuren S, Groothuis-Oudshoorn K. mice: Multivariate Imputation by Chained Equations in R. Journal of Statistical Software. 2011;45(3):67.
Available online at: www.jccm.ro

13. Zhang $\mathbf{C}-\mathrm{H}$. Nearly unbiased variable selection under minimax concave penalty. Ann Statist. 2010;38(2):894-942.

14. Breheny P, Huang J. COORDINATE DESCENT ALGORITHMS FOR NONCONVEX PENALISED REGRESSION, WITH APPLICATIONS TO BIOLOGICAL FEATURE SELECTION. Ann Appl Stat. 2011;5(1):232-253.

15. Truog RD, Mitchell C, Daley GQ. The Toughest Triage Allocating Ventilators in a Pandemic. N Engl J Med. 2020 May 21;382(21):1973-1975.

16. Kózka M, Sega A, Wojnar-Gruszka K, Tarnawska A, Gniadek A. Risk Factors of Pneumonia Associated with Mechanical Ventilation. Int J Environ Res Public Health. 2020;17(2):656.

17. Barrett B, Pamphile S, Yang F, et al. Inflammatory markers are poorly predictive of clinical outcomes among hospitalised patients with COVID-19. Am J Emerg Med. 2021 Aug; 46: 595598.

18. Ramachandran P, Onukogu I, Ghanta S, et al. Gastrointestinal Symptoms and Outcomes in Hospitalized Coronavirus Disease 2019 Patients. Digestive Diseases. 2020;38(5):373-379.

19. Henry BM, de Oliveira MHS, Benoit J, Lippi G. Gastrointestinal symptoms associated with severity of coronavirus disease 2019 (COVID-19): a pooled analysis. Intern Emerg Med. 2020;15(5):857-859.

20. Ranieri VM, Rubenfeld GD, Thompson BT, et al. Acute respiratory distress syndrome: the Berlin Definition. Jama. 2012;307(23):2526-2533. 